\title{
The effect of low dose ketamine on postoperative quality of recovery in patients undergoing breast cancer surgery: a randomized, placebo controlled trial
}

\section{Yuan Han}

The Affiliated Hospital of Xuzhou Medical University

Zijian Zhao

Xuzhou Medical University

\section{Qiqi Xu}

Xuzhou Medical University

Yao Chen

Xuzhou Medical University

Chen Liu

Xuzhou Medical University

Fangfang Zhang

Xuzhou Medical University

\section{Xing Gao}

Xuzhou Medical University

\section{Huilian Guan}

Xuzhou Medical University

\section{Mingsheng Dai}

Xuzhou Medical University

He Liu

The Affiliated Hospital of Xuzhou Medical University

Junli Cao ( $\square$ junlicao0310@163.com )

The affiliated hospital of Xuzhou Medical University https://orcid.org/0000-0003-1868-9603

\section{Research article}

Keywords:

Posted Date: August 26th, 2019

DOI: https://doi.org/10.21203/rs.2.12880/v1 
License: (c) (i) This work is licensed under a Creative Commons Attribution 4.0 International License. Read Full License 


\section{Abstract}

Background: Intraoperative low dose ketamine has been widely studied for postoperative multimodal analgesia. However, the effect of low dose ketamine on postoperative quality of recovery in female patients undergoing breast cancer surgery has not been studied. The primary aim of this study was to assess the effects of low dose ketamine on postoperative quality of recovery in these patients using the 40-Item Quality of Recovery (QoR-40) scale. The second aim of this study was to evaluate the effects of intraoperative infusion of low-dose ketamine on chronic postoperative surgical pain (CPSP). Methods: In this prospective, randomized, double-blind trial, 100 patients planned for modified radial mastectomy were randomly assigned to one of two groups: control group (group C) and ketamine group (group K). After induction of anesthesia and before surgical incision, a bolus dose of $0.5 \mathrm{mg} / \mathrm{kg}$ ketamine or an equivalent volume of normal saline was injected followed by $0.25 \mathrm{mg} / \mathrm{kg} / \mathrm{h}$ ketamine or normal saline infusions until skin closure. Postoperative recovery profiles, including the QoR-40 scores on postoperative day 1 , numeric rating scale (NRS), and douleur neuropathique 4 (DN-4) questionnaire at postoperative month 3 were evaluated. In addition, patient characteristics, perioperative parameters, and safety outcomes were assessed. Results: Global QoR-40 scores on postoperative day 1 were not significantly different between both groups $(p=0.139)$. Of the five dimensions, emotional state and pain scores were significantly higher in group $K$ than in group $C(p=0.044$ and 0.023 , respectively). At postoperative month 3 , CPSP and neuropathic pain (NP) were not significantly different between groups $(p=0.259$ and 0.959 , respectively). The incidence of safety outcomes were similar between groups. Conclusion: Low dose ketamine did not improve overall quality of recovery or chronic pain in patients undergoing breast cancer surgery.

\section{Background}

Breast cancer is one of the most common cancers in women globally. Advances in diagnosis and treatment have significantly improved breast cancer survival rates. ${ }^{1}$ To facilitate reintegration into society, effort has been made to improve the speed and quality of comprehensive recovery. Adequate control of acute pain after modified radial mastectomy is crucial for recovery, but chronic postsurgical pain (CPSP) after mastectomy is often underestimated and poorly recognized. ${ }^{2}$ Indeed, the incidence of chronic and neuropathic pain after modified radical mastectomy is relatively high across surgery types. It often has a neuropathic component and continues to plague patients. ${ }^{3}$ Thus, identifying effective treatments to promote patient recovery and reduce chronic pain is critical for breast cancer patients to reintegrate into society.

The use of multimodal analgesia for relieving post-operative pain can improve analgesia and reduce the incidence of opioid-related adverse events. Ketamine, an N-methyl-D-aspartate (NMDA) receptor antagonist, is increasingly used as an alternative for pain control during the acute perioperative period. A randomized, blinded trial reported that intraoperative ketamine significantly reduced morphine consumption after lumbar fusion surgery in opioid-dependent patients. ${ }^{4}$ Further, low-dose $(<1 \mathrm{mg} / \mathrm{kg})$ 
ketamine may be beneficial for acute and chronic post-operative pain management. ${ }^{5,6}$ Ketamine interaction with NMDA receptors is involved in opioid-induced anti-hyperalgesia. Ketamine suppresses the induction of nitric oxide synthase activity and protein expression by endotoxins, and has protective antiinflammatory effects against sepsis. ${ }^{7}$

Modern anesthesia management has led to greater emphasis on post-operative outcomes. Technical measurements encompass assessment of time to awakening, length of hospital stay, nausea, vomiting, and pain control. The 40-Item Quality of Recovery Questionnaire (QoR-40) is sensitive to changes in health status and provides an overall health status measure after surgery and anesthesia. It is considered the best instrument for evaluating the complex and multidimensional process of postoperative recovery in the general surgical population. ${ }^{8,9}$

Although the effects of low-dose ketamine on the quality of recovery after laparoscopic cholecystectomy have been investigated, ${ }^{10} \mathrm{few}$ studies have assessed how low-dose ketamine affects quality of recovery and chronic pain after radial mastectomy. We hypothesized that low-dose ketamine would enhance patients' quality of recovery following modified radial mastectomy under total intravenous anesthesia. This study's primary objective was to assess the effects of low-dose ketamine on quality of recovery in patients undergoing modified radial mastectomy under remifentanil-propofol-based anesthesia using the QoR-40 questionnaire. The second objective was to evaluate the effects of intraoperative infusion of low-dose ketamine on mastectomy-induced chronic pain.

\section{Methods}

\section{Subjects}

We performed a prospective, randomized, double-blind clinical trial, which was approved by the Clinical Research Ethics Committee of the Affiliated Hospital of Xuzhou Medical University, Jiangsu, China (Certification No.XYFY2018-KL058-01, approved date: August 27, 2018) and was registered before patient enrollment at https://www.clinicaltrials.gov (number: NCT03676114; principle investigator: J. L. C.; date of Registration: September 18, 2018). Written informed consent was obtained from all patients participating in the trial. This manuscript adheres to all applicable CONSORT guidelines.

Female patients aged 20-65 years old and American Society of Anesthesiologists physical status I or II scheduled to undergo breast cancer surgery (modified radical mastectomy or wide local excision and axillary node dissection, including sentinel node mapping or clearance) under general anesthesia were enrolled between September 2018 and February 2019. Exclusion criteria included: unstable hypertension, history of heart disease, hepatic or renal dysfunction, undergoing chemotherapy before surgery, history of chronic pain or chronic use of analgesics, mental illness or inability to cooperate with investigators, and/or history of ketamine allergy. 


\section{Intervention}

Patients were randomly assigned to one of two groups based on computer generated codes that were maintained in sequentially numbered opaque envelopes: control group (group C) and ketamine group (group K). On the morning of surgery day, a responsible anesthesiologist who was not involved in patient evaluation opened the envelope and prepared either $0.5 \%$ ketamine or normal saline in $20 \mathrm{~mL}$ syringes, then labeled them as "study drug" for double-blind purposes. After induction of anesthesia and before surgical incision, a bolus dose of $0.5 \mathrm{mg} / \mathrm{kg}$ ketamine or an equivalent volume of normal saline was injected via a reliable intravenous catheter, followed by $0.25 \mathrm{mg} \cdot \mathrm{kg}^{-1} \cdot \mathrm{h}^{-1}$ ketamine or normal saline infusions until skin closure.

The ketamine diluents were mixed to concentrations of $5 \mathrm{mg} / \mathrm{mL}$ to ensure that all drugs were infused at a bolus of $0.1 \mathrm{~mL} / \mathrm{kg}$ followed by $0.05 \mathrm{~mL} \cdot \mathrm{kg}^{-1} \cdot \mathrm{h}^{-1}$ thereafter. None of the investigators involved in patient management or data collection were aware of the group assignments.

\section{Anesthetic Management}

All patients fasted for at least 6-8 hours before surgery. After entering the operating room, the venous access was opened. Heart rate (HR), blood pressure (BP), pulse oxygen saturation $\left(\mathrm{SpO}_{2}\right)$, and electrocardiogram (ECG) were routinely monitored. Anesthesia was induced with midazolam $(0.05$ $\mathrm{mg} / \mathrm{kg})$, etomidate $(0.3 \mathrm{mg} / \mathrm{kg})$, sufentanil $(0.5 \mathrm{ug} / \mathrm{kg})$ and rocuronium $(0.6 \sim 1 \mathrm{mg} / \mathrm{kg})$. The tracheal intubation was performed under the visual laryngoscope, and the ventilator was connected with the tidal volume of $8 \mathrm{ml} / \mathrm{kg}$. The respiratory rate was adjusted to maintain the end-expiratory $\mathrm{CO}_{2}$ partial pressure at 35-45 mmHg. Propofol (4-6 $\left.\mathrm{mg} \cdot \mathrm{kg}^{-1} \cdot \mathrm{h}^{-1}\right)$ and remifentanil $\left(0.3 \mu \mathrm{g} \cdot \mathrm{kg}^{-1} \cdot \mathrm{min}^{-1}\right)$ were used for anesthesia maintenance. Depth of anesthesia was titrated by adjusting the dose of propofol and remifentanil to maintain mean arterial pressure and heart rate within $20 \%$ of the pre-induction values. According to the surgical situation, the muscle relaxant is added, and no volatile anesthetic is used for induction and maintenance. All anesthetics were discontinued at the end of the suture. The tracheal tube can be removed when the patient regained consciousness and the desired tidal volume was achieved. The patients were then transferred to the postanesthetic care unit (PACU). The patient-controlled analgesia (PCA) pump was not routinely placed after the operation, and the doctor in the ward gave an appropriate amount of flurbiprofen axetil or dezocine according to the patient's pain complaint.

\section{Primary Outcome}

The primary end point of the study was the global QoR-40 score on postoperative day 1 (POD 1).

\section{Secondary Outcomes}


The secondary end points included the effects of intraoperative ketamine on CPSP at 3 months after modified radical mastectomy. Simultaneously, vital signs during the anesthetic period, including heart rate, peripheral oxygen saturation, and mean arterial pressure were measured and recorded at three time points (10 min after ketamine infusion [T1]; moment of extubation [T2]; 10 min after extubation [T3]). Other perioperative data such as adverse reactions to ketamine, postoperative remedial analgesia and the number of patients who received chemotherapy and radiotherapy were also recorded.

\section{Outcome Assessment}

A researcher who was unaware of the group assignments visited each patient to administer the QoR-40 survey the day before surgery and on POD1.

QoR-40 is a self-scoring questionnaire that examines the conditions associated with patients' recovery, including five dimensions: physical comfort, emotional state, physical independence, psychological support and pain, for a total of 40 items. The scores of each item are added together as the final total score. The lowest score is 40 points and the highest score is 200 points, the higher the score, the better the patients recover.The QoR-40 survey has been widely used and validated for patients undergoing a variety of surgical procedures.

Three months postoperatively, a phone-based questionnaire was conducted to assess the presence or absence of persistent pain, intensity, and character of pain. Prevalence of pain was assessed dichotomously with yes/no questions. Intensity was assessed using NRS, where we interpreted 1-3 as light pain, 4-6 as moderate pain, and $7-10$ as severe pain. Chronic pain was also assessed using the douleur neuropathique $4(\mathrm{DN}-4)$ questionnaire, whereby scores of $\geq 4$ were indicative of pain with neuropathic characteristics. ${ }^{11}$

\section{Statistical Analysis}

All continuous variables data were assessed for normality using the Kolmogorov-Smirnov tests, and Levene test was used to assess equality of variances. Group comparisons were made using two independent sample t-tests for continuous variables with a normal distribution, the Mann-Whitney $U$ test for continuous variables with a non-normal distribution, or the chi-square test or Fisher's exact test for dichotomous and ranked data. A value of $p<0.05$ was used for all comparisons. All statistical analyses were completed using SPSS, version 22.0 (IBM, New York, NY).

According to previous studies, a QoR-40 score of 10 or more differences was considered to be a clinically significant improvement or worsening of recovery quality. Based on our preliminary data, the variability (SD) of QoR-40 scores on the first day after breast cancer surgery was 15 . The estimated sample size was 37 patients per group with a power of $80 \%$ at an a level of 0.05 . We enrolled a final total of 50 patients per group, allowing for a $30 \%$ drop-out rate. 


\section{Results}

Among 205 patients assessed for study participation between September 2018 and February 2019, 110 patients who provided consent were enrolled. A total of 95 patients were excluded from the study; among them, 73 patients did not meet inclusion criteria, 10 patients satisfied the exclusion criteria, and 12 patients declined to participate. A total of 100 patients were randomly assigned to the different groups, as seven patients cancelled the operation and three patients withdrew consent; 49 patients were allocated to group C, and 48 patients were allocated to group K. Primary outcomes analysis consisted of 49 patients in group $\mathrm{C}$ and 48 patients in group $\mathrm{K}$, as one patient changed operation in group $\mathrm{C}$, one patient changed operation Because the pathology report is uncertain, and one patient dropped out for physician's reasons in group K. At 3 months after surgery, seven patients had wound infection, and four patients were lost to follow-up. The final analysis consisted of 44 patients in group $\mathrm{C}$ and 42 patients in group K (Figure 1). Patients' characteristics are shown in Table 1. There were no statistically significant differences in baseline data between the two groups.

\section{Primary Outcomes}

The global QoR-40 scores and subscores of each dimension for the two groups are shown in Table 2. There were no differences between groups in global QoR-40 scores on POD1 ( $p=0.139)$. Of the five dimensions, emotional state and pain scores were significantly higher in group $\mathrm{K}$ than in group $\mathrm{C}(p=$ 0.044 and 0.023 , respectively). No differences were detected in preoperative data in both groups. Scores for the three other dimensions on POD 1 were not significantly different between group $\mathrm{C}$ and group $\mathrm{K}$.

\section{Secondary Outcomes}

Other surgery-related data are presented in Table 3. Vital signs recorded during surgery, heart rate, peripheral oxygen saturation, and mean arterial pressure recorded at three time points did not exhibit significant differences between groups. Propofol and remifentani consumption were not significantly different between groups ( $p=0.952$ and 0.176 , respectively). There were no differences in emergence time and duration of PACU stay ( $p=0.517$ and 0.848 , respectively) between groups. The number of analgesics required by patients was similar between groups. Safety outcomes, such as delirium, nightmares, nausea, vomiting, and drowsiness were not significantly different between groups. No hallucinations or chills were observed in any of the participants (Table 4).

There ware no statisticaliy differences between groups in patients who received chemotherapy and radiotherapy (Table 5). We evaluated the role of ketamine on chronic pain and neuropathic pain among female breast cancer patients after mastectomy. The incidence of CPSP (NRS $₫ 0)$ and neuropathic pain (DN-4 $\geq 4)$ after modified radical mastectomy were not significantly different between groups $(p=0.259$ and 0.959 , respectively). The ratio of neuropathic pain (NP) and CPSP was $73.9 \%$ in group C and $59.3 \%$ in group $\mathrm{K}$, but the ratio was not significantly different between groups $(p=0.276)$. There were no differences in proportion of moderate or severe pain (NRS $\varangle 3)(p=0.685)$. As ketamine has anti- 
hyperalgesic effects, we listed the last item in DN-4 (allodynia) separately, but no significant difference was observed $(p=0.885)$.

\section{Discussion}

In this study, we did not observe any significant difference in patients who received continuous intraoperative low dose ketamine and control patients who did not receive ketamine, in terms of global core of QoR or chronic pain scores following mastectomy under total intravenous anesthesia. However, among the five dimensions, emotional state and pain scores were significantly higher in the group that received ketamine. To our knowledge, the present study is the first to report the effects of continuous intraoperatively administered low dose ketamine on quality of recovery using the QoR-40 survey and chronic pain with DN-4 among Asian female breast cancer patients after radial mastectomy.

When defining the quality of post-operative recovery, other aspects relating to post-operative recovery alongside pain control must be considered. For instance, patients' emotional and psychological state, physical comfort, and physical independence should be considered. The global QoR-40 is the first established and verified clinical tool for measuring functional recovery in the immediate postoperative period. Its scoring system contains five different dimensions and assesses pain as well as aforementioned aspects of recovery. While it is suggested that a minimum 10-point difference represents clinically relevant improvement in quality of recovery, ${ }^{12}$ a recent study reported that a change of $6.3 \mathrm{can}$ be the minimum clinically important difference. ${ }^{13}$ In the present study, median QoR-40 scores in group $\mathrm{K}$ were approximately 3 higher than those in group $C$ at POD1, but this difference was not statistically significant. Nevertheless, as the median difference in QoR-40 between the two groups was only 3 and the minimum clinically important difference is at least 6.3 , expanding the sample size to observe a greater effect may be worthwhile and should be considered in future studies. Further statistical analysis of each QoR-40 dimension may offer additional valuable information. Thus, we conducted statistical analysis of each dimension of QoR-40 between groups. This revealed that the ketamine group has higher emotional state and pain scores than those in the control group. These data indicate that ketamine may help to improve patients' mood and analgesia but may not be beneficial for other aspects of the recovery.

By activating NMDA receptors, persistent nociceptive input can lead to pain sensitization. The mechanism of ketamine-induced anti-hyperalgesia is predominantly by noncompetitive antagonism of the NMDA receptor. This promotes structural synaptic connectivity thereby leading to prolonged antidepressant effects. ${ }^{14}$ Opioid-induced hyperalgesia $(\mathrm{OIH})$ refers to the long-term application of opioid analgesics, and the damage caused by peripheral tissue damage or inflammation has a strong nociceptive response to noxious stimuli, and NMDA receptors play a key role in OIH. As an NMDA receptor antagonist, ketamine is thought to prevent or reduce $\mathrm{OIH}$, thus reducing the use of analgesics and the adverse effects of $\mathrm{OlH}$. Ketamine was also shown to reduce hyperalgesia, prevent opioid tolerance in animals, ${ }^{15}$ reduce morphine resistance in humans, ${ }^{16}$ and decrease morphine consumption after 
transthoracic lung and heart surgery. ${ }^{17} \mathrm{~A}$ high-quality systematic review reported that perioperative use of ketamine could reduce opioid consumption and time to the first analgesic request. ${ }^{18}$

The incidence of post-mastectomy chronic pain is substantially higher than in other types of surgery, ranging from 20 to $50 \% .3,19,20$ The presence of chronic pain affects the quality of recovery from surgery trauma and results in poor quality of life. The NRS is used for assessing the quality and intensity of chronic pain, and the $\mathrm{DN}-4$ is the most widely used tool for screening neuropathic pain. ${ }^{11}$ Due to the predominance of the neuropathic component of pain ${ }^{21}$ which is linked to the activation of NMDA receptors, it is believed that drugs blocking NMDA receptors may be effective in the treatment of patients with post-mastectomy chronic pain syndrome. However, in the present study, we did not observe any difference in the incidence of CPSP or NP between group $\mathrm{K}$ and group $\mathrm{C}$.

There are several reasons for the fall of ketamine treatment in patients' recovery and chronic pain. First, the timing, dose, and type of infusion of ketamine administration are critical factors modulating its effects. Subanesthetic doses of ketamine have sufficient analgesic efficacy by inhibiting NMDA receptormediated pain facilitation following noxious stimuli. In contrast, high doses of ketamine may lead to more severe side effects, such as the emergence of hallucinations and nightmares, thereby limiting its use. $^{6}$ Since the clinical analgesia block provided by a bolus injection of ketamine at a dose of $0.15 \mathrm{mg} / \mathrm{kg}$ would last for less than 5 mins, continuous intravenous infusion of ketamine throughout the operation and even into the recovery period would be needed for greater effectiveness. ${ }^{22}$ Besides, subanesthetic ketamine administered only intraoperatively is unlikely to cause major psychomimetic adverse effects, while postoperative infusions are associated with limited and reversible psychomimetic adverse effects. ${ }^{23}$ So in the present article, considering the side effects caused by higher and long-term administration of ketamine, we administered a bolus dose of subanesthetic ketamine followed by continuous intravenous infusion only until the end of the surgery. The dosage we selected in this article mainly referred to the study in rectal adenocarcinoma surgery. ${ }^{24}$ But we did not observed similar positive results, considering surgery types differ, especially the sensory nerve injury or damage is small in rectal surgery, the pathophysiology of chronic pain is not the same. In a future study, the dose of ketamine or the length of infusion may have to be increased to achieve better outcomes. Another reason may be that other analgesics, such as nonsteroidal anti-inflammatory drugs and glucocorticoids, were not restricted between groups, which may also have the ability to block NMDA receptors and reduce the differences in recovery quality and analgesia effects between groups. Thus, when the administration of multimodal perioperative analgesia is adequate, ketamine may become ineffective or even unnecessary.

There are several limitations of our study. First, we only conducted the trial on healthy female participants, thus we cannot generalize our findings to the general population, including those with severe illness. Second, we excluded subjects with a history of chronic pain or those taking analgesics before surgery as this population would introduce a bias in the subject sample in this prospective randomized clinical trial. A recent study has highlighted a possible role for ketamine in opioid-dependent chronic pain patients. ${ }^{25}$ Patients in ketamine group had significantly reduced postoperative morphine consumption 
and better outcomes at 6 months, so the effect of ketamine in opioid-dependent patients is worthing exploring. Third, we did not evaluate whether the treatment would benefit quality of recovery beyond $24 \mathrm{~h}$, although ketamine did not significantly facilitate patients' recovery one day after surgery. Finally, although this study was adequately powered to detect a difference in the primary outcome, it may not have had sufficient power to detect differences in chronic pain control. Further studies should evaluate higher doses or extend the administration of ketamine into the recovery period in both male and female patients.

\section{Conclusion}

In conclusion, intravenous ketamine during surgery did not promote superior overall quality of recovery by preventing physiologic deterioration related to anesthesia and mastectomy. Further, it did not improve chronic pain related to surgery. Therefore, based on this study, intravenous low dose ketamine did not benefit female patients overall quality of recovery or chronic pain undergoing breast cancer surgery.

\section{List Of Abbreviations}

ASA

American Society of Anesthesiologists

CPSP

Postoperative surgical pain

$\mathrm{DN}-4$

Douleur neuropathique 4

$\mathrm{HR}$

Heart rate

MAP

Mean arterial pressure

NMDA

$\mathrm{N}$-methyl-D-aspartate

NP

Neuropathic pain 
NRS

Numerical rating scale

$\mathrm{OIH}$

Opioid-induced hyperalgesia

PACU

Postanesthetic care unit

QoR-40

40-Item quality of recovery

SD

Standard deviation

$\mathrm{SpO} 2$

Peripheral oxygen saturation

\section{Declarations}

\section{Ethics approval and consent to participate:}

The trial was approved by the Clinical Research Ethics Committee of the Affiliated Hospital of Xuzhou Medical University, Jiangsu, China (Certification No.XYFY2018-KL058-01, approved date: August 27, 2018). Written informed consent was obtained from all patients participating in the trial.

\section{Consent for publication:}

Not applicable

\section{Availability of data and materials:}

The datasets generated and/or analyzed during the current study will be available from the corresponding author on reasonable request. 
National Natural Science Foundation of China (81720108013, 81571059); China Postdoctoral Science Foundation Funded Project (2018T110556). The funding bodies played the roles in the design of the study and collection, analysis, and interpretation of data and in writing the manuscript.

\section{Competing Interests:}

None

\section{Author's contributions:}

JLC: This author designed the study, critical review of the manuscript, approves the final version and is accountable for the work.

$\mathrm{YH}$ : This author designed the study, analyze the data, draft the manuscript, and approve the final version of the manuscript.

ZJZ: This author helped designed the study and analyze the data, draft the manuscript, and approve the final version of the manuscript.

QQX: This author helped designed the study and analyze the data, draft the manuscript, and approve the final version of the manuscript.

YC: This author helped designed the study and analyze the data, draft the manuscript, and approve the final version of the manuscript.

CL: This author helped recruitment, conduct the randomize, and critical review of the manuscript.

FFZ: This author helped recruitment, conduct the blind, and critical review of the manuscript.

XG: This author helped collect the operative data, and critical review of the manuscript.

HLG: This author helped search literature, collect the operative data, and critical review of the manuscript.

MSD: This author helped search literature, collect the follow-up data, and critical review of the manuscript.

HL: This author helped search literature, collect the follow-up data, and critical review of the manuscript.

All authors have read and approved the final manuscript.

\section{Acknowledgements:}


None

\section{References}

[1]Wang Y C, Wei L J, Liu J T, et al. Comparison of Cancer Incidence between China and the USA. Cancer Biol Med, 2012,9(2):128-132.

[2]Kehlet $\mathrm{H}$, Jensen T S, Woolf C J. Persistent postsurgical pain: risk factors and prevention. Lancet, 2006,367(9522):1618-1625.

[3]Haroutiunian S, Nikolajsen L, Finnerup N B, et al. The neuropathic component in persistent postsurgical pain: a systematic literature review. Pain, 2013,154(1):95-102.

[4]Nielsen R V, Fomsgaard J S, Siegel H, et al. Intraoperative ketamine reduces immediate postoperative opioid consumption after spinal fusion surgery in chronic pain patients with opioid dependency: a randomized, blinded trial. Pain, 2017,158(3):463-470.

[5]Kwok R F, Lim J, Chan M T, et al. Preoperative ketamine improves postoperative analgesia after gynecologic laparoscopic surgery. Anesth Analg, 2004,98(4):1044-1049.

[6]McNicol E D, Schumann R, Haroutounian S. A systematic review and meta-analysis of ketamine for the prevention of persistent post-surgical pain. Acta Anaesthesiol Scand, 2014,58(10):1199-1213.

[7]Yoon S H. Concerns of the anesthesiologist: anesthetic induction in severe sepsis or septic shock patients. Korean J Anesthesiol, 2012,63(1):3-10.

[8]Myles P S, Weitkamp B, Jones K, et al. Validity and reliability of a postoperative quality of recovery score: the QoR-40. Br J Anaesth, 2000,84(1):11-15.

[9]Gornall B F, Myles P S, Smith C L, et al. Measurement of quality of recovery using the QoR-40: a quantitative systematic review. Br J Anaesth, 2013,111(2):161-169.

[10]Moro E T, Feitosa I, de Oliveira R G, et al. Ketamine does not enhance the quality of recovery following laparoscopic cholecystectomy: a randomized controlled trial. Acta Anaesthesiol Scand, 2017,61(7):740748.

[11]Bouhassira D, Attal N, Alchaar $\mathrm{H}$, et al. Comparison of pain syndromes associated with nervous or somatic lesions and development of a new neuropathic pain diagnostic questionnaire (DN4). Pain, 2005,114(1-2):29-36.

[12]Myles P S, Hunt J O, Nightingale C E, et al. Development and psychometric testing of a quality of recovery score after general anesthesia and surgery in adults. Anesth Analg, 1999,88(1):83-90. 
[13]Myles P S, Myles D B, Galagher W, et al. Minimal Clinically Important Difference for Three Quality of Recovery Scales. Anesthesiology, 2016,125(1):39-45.

[14]Sleigh J, Harvey M, Voss L, et al. Ketamine - More mechanisms of action than just NMDA blockade. Trends in Anaesthesia \& Critical Care, 2014,4(2-3):76-81.

[15]Stubhaug A, Breivik H, Eide P K, et al. Mapping of punctuate hyperalgesia around a surgical incision demonstrates that ketamine is a powerful suppressor of central sensitization to pain following surgery. Acta Anaesthesiol Scand, 1997,41(9):1124-1132.

[16]Atangana R, Ngowe N M, Binam F, et al. Morphine versus morphine-ketamine association in the management of post operative pain in thoracic surgery. Acta Anaesthesiol Belg, 2007,58(2):125-127.

[17]Nesher N, Serovian I, Marouani N, et al. Ketamine spares morphine consumption after transthoracic lung and heart surgery without adverse hemodynamic effects. Pharmacol Res, 2008,58(1):38-44.

[18]Laskowski K, Stirling A, McKay W P, et al. A systematic review of intravenous ketamine for postoperative analgesia. Can J Anaesth, 2011,58(10):911-923.

[19]Smith W C, Bourne D, Squair J, et al. A retrospective cohort study of post mastectomy pain syndrome. Pain, 1999,83(1):91-95.

[20]Macdonald L, Bruce J, Scott N W, et al. Long-term follow-up of breast cancer survivors with postmastectomy pain syndrome. Br J Cancer, 2005,92(2):225-230.

[21]Jung B F, Ahrendt G M, Oaklander A L, et al. Neuropathic pain following breast cancer surgery: proposed classification and research update. Pain, 2003,104(1-2):1-13.

[22]Himmelseher S, Durieux M E. Ketamine for perioperative pain management. Anesthesiology, 2005,102(1):211-220.

[23]Steven P, Anuj Bhatia, Asokumar Buvanendran, et al. Consensus Guidelines on the Use of Intravenous Ketamine Infusions for Chronic Pain Management From the American Society of Regional Anesthesia and Pain Medicine, the American Academy of Pain Medicine, and the American Society of Anesthesiologists. Reg Anesth Pain Med, 2018, 43: 00-00

[24] De Kock M, Lavand'homme P, Waterloos H. 'Balanced analgesia' in the perioperative period: is there a place for ketamine? Pain, 2001, 92:373-380

[25] Nielsen RV, Fomsgaard JS, Siegel H, et al. Intraoperative ketamine reduces immediate postoperative opioid consumption after spinal fusion surgery in chronic pain patients with opioid dependency: a randomized, blinded trial. Pain, 2017, 158:463-470.

\section{Tables}


Table 1. Patients' Characteristics

\begin{tabular}{cccc}
\hline & Group C (n=50) & Group K (n=50) & $p$ value \\
\hline Age(y) & $50.7(8.3)$ & $50.4(8.5)$ & 0.859 \\
Height(cm) & $159.6(4.6)$ & $159.3(4.3)$ & 0.721 \\
Weight(kg) & $60.0(8.0)$ & $62.1(8.5)$ & 0.210 \\
BMI $\left(\mathrm{kg} / \mathrm{m}^{2}\right)$ & $23.6(2.9)$ & $24.4(3.0)$ & 0.138 \\
ASA physical status & & & 0.640 \\
$\square$ & $39(78 \%)$ & $37(74 \%)$ & \\
$\square$ & $11(22 \%)$ & $13(26 \%)$ & \\
Operation time (min) & $101.9(44.0)$ & $104.8(40.3)$ & 0.730 \\
Anesthesia time (min) & $129.5(45.5)$ & $136.6(39.7)$ & 0.406 \\
Preoperative pain scores & $0(0,0)$ & $0(0,1.25)$ & 0.246 \\
NRS at rest & $0(0,2)$ & $0(0,2)$ & 0.483 \\
NRS when moving & & & \\
\hline
\end{tabular}

Data are presented as mean (SD, standard devition) $\llbracket$ median (IQR,interquartile range) or numbers (proportion, $\%)$.

Abbreviations: Group C, control group; Group K, ketamine group; BMI, Body Mass Index; ASA, American Society of Anesthesiologists; NRS, numeric rating scale.

Table 2. The Global QoR-40 Scores Between Groups 


\begin{tabular}{|c|c|c|c|}
\hline & Group C $(n=49)$ & Group K $(n=48)$ & $P$ value \\
\hline Global QoR-40 & & & \\
\hline Preoperative & $195(187,197)$ & $193(189,196.5)$ & 0.750 \\
\hline $\begin{array}{c}\text { POD1 } \\
\text { QoR-40 dimensions }\end{array}$ & $170(165,177)$ & $173(169,177)$ & 0.139 \\
\hline Emotional state & & & \\
\hline Preoperative & $43(40,45)$ & $43(41,44)$ & 0.826 \\
\hline $\begin{array}{c}\text { POD1 } \\
\text { Physical comfort }\end{array}$ & $41(39,43)$ & $42(40,44)$ & $0.044^{\mathrm{a}}$ \\
\hline Preoperative & $58(55,59)$ & $57(55,59)$ & 0.790 \\
\hline $\begin{array}{c}\text { POD1 } \\
\text { Psychological support }\end{array}$ & $52(49,56)$ & $53(50.5,55)$ & 0.452 \\
\hline Preoperative & $35(34,35)$ & $35(34,35)$ & 0.580 \\
\hline $\begin{array}{c}\text { POD1 } \\
\text { Physical independence }\end{array}$ & $35(34,35)$ & $35(34,35)$ & 0.239 \\
\hline Preoperative & $25(25,25)$ & $25(25,25)$ & 0.335 \\
\hline POD1 & $12(11,13)$ & $12(10,14)$ & 0.311 \\
\hline Preoperative & $34(33,35)$ & $34(33.5,35)$ & 0.831 \\
\hline POD1 & $31(28,32)$ & $31.5(30,33)$ & $0.023^{a}$ \\
\hline
\end{tabular}

Data are presented as median (IQR,interquartile range).

Abbreviations: Group C, control group; Group K, ketamine group; QoR-40, quality of recovery 40; POD1, postoperation day 1.

${ }^{\mathrm{a}} p<.05$, Mann-Whitney U test.

Table 3. Perioperative Parameters 


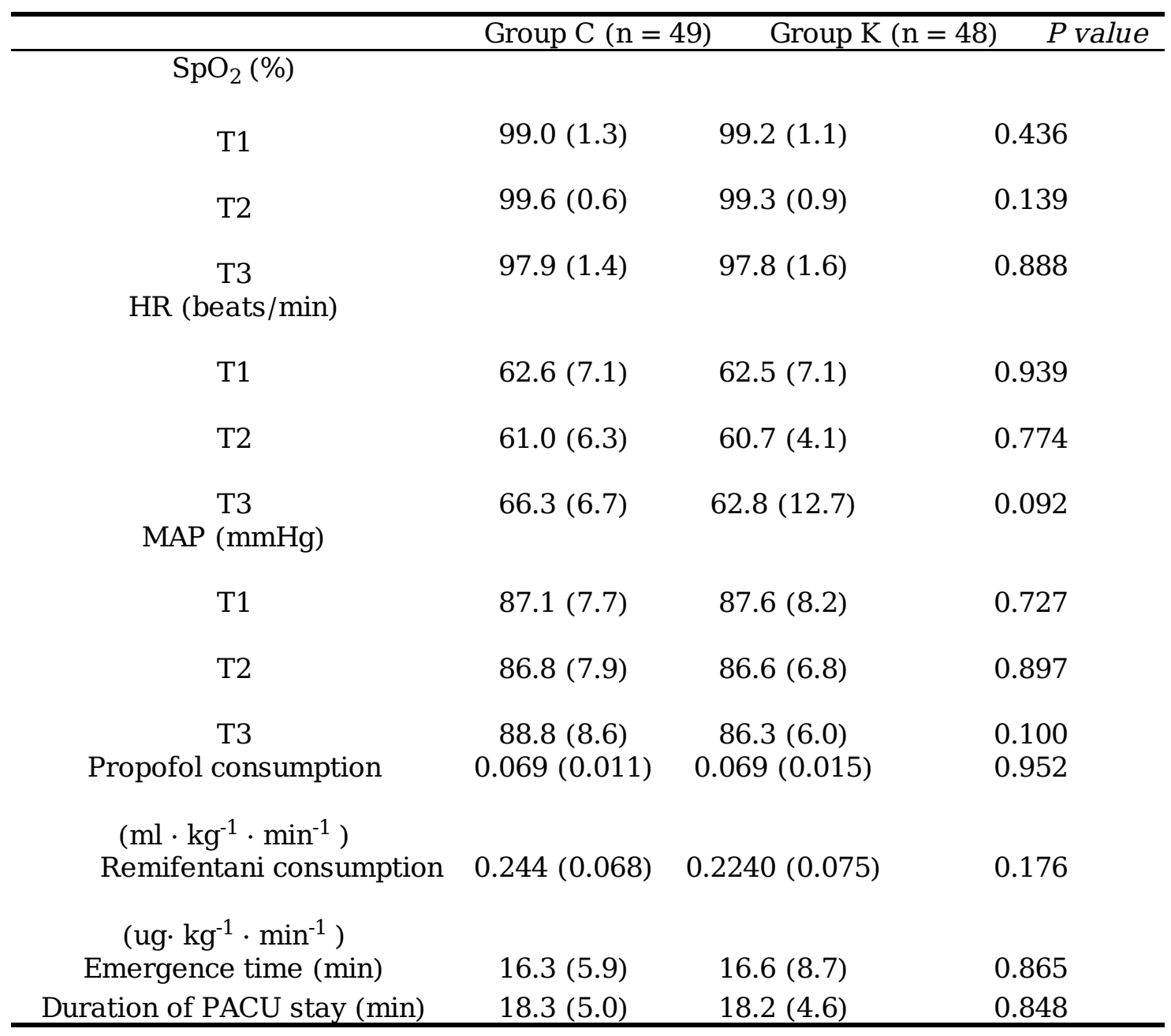

Data are presented as mean (SD, standard devition).

Abbreviations: Group C, control group; Group K, ketamine group; Spo2, peripheral oxygen saturation; HR, heart rate; MAP, mean arterial blood pressure;PACU, postanesthetic care unit;T1, 10 min after ketamine infusion; T2, moment of extubation;T3, 10 min after extubation; emergence time, from discontinuation of anesthetics to extubation.

Table 4. Postoperative acute pain data and safety outcomes 


\begin{tabular}{|c|c|c|c|}
\hline & Group C $(n=49)$ & Group K $(\mathrm{n}=48)$ & $p$ value \\
\hline \multicolumn{4}{|l|}{ Pain at rest } \\
\hline $4 \mathrm{~h}$ & $2(0,4)$ & $2(0,4)$ & 0.751 \\
\hline $24 \mathrm{~h}$ & $2(2,4)$ & $2(1.25,3)$ & 0.810 \\
\hline $48 \mathrm{~h}$ & $2(1,3)$ & $2(0,2)$ & 0.701 \\
\hline Pain when moving & $3(1,5)$ & $3(1.25,4)$ & 0.491 \\
\hline $4 \mathrm{~h}$ & $3(2,5)$ & $3(2.25,4.75)$ & 0.895 \\
\hline $24 \mathrm{~h}$ & $2(1,3.5)$ & $2(2,3)$ & 0.795 \\
\hline \multicolumn{4}{|l|}{$48 \mathrm{~h}$} \\
\hline Analgesics requirement (number) & 3 (6.1\%) & $2(4.2 \%)$ & 1.000 \\
\hline Nightmares & $1(2.0 \%)$ & $1(2.1 \%)$ & 1.000 \\
\hline Delirium & $0(0 \%)$ & 1 (2.1\%) & 1.000 \\
\hline Nausea & 26 (53.1\%) & 25 (52.1\%) & 0.838 \\
\hline Vomitting & 14 (28.6\%) & 15 (31.3\%) & 0.824 \\
\hline Drowsiness & 7 (14.3\%) & 9 (18.8\%) & 0.584 \\
\hline
\end{tabular}

Data are presented as median (IQR,interquartile range) or numbers (proportion, \%).

Abbreviations: Group C, control group; Group K, ketamine group.

Hallucinations and chill are not found in this study.

Table 5. Postoperative chronic pain data

\begin{tabular}{cccc}
\hline & Group C $(\mathrm{n}=44)$ & Group K (n=42) & $P$ value \\
\hline CPSP (NRSø0) & $23(52.3 \%)$ & $27(64.3 \%)$ & 0.259 \\
Moderate or severe pain (NRS₫3) & $5(11.4 \%)$ & $6(14.3 \%)$ & 0.685 \\
Neuropathic pain & $17(38.6 \%)$ & $16(38.1 \%)$ & 0.959 \\
Tactile pain & $10(22.7 \%)$ & $9(21.4 \%)$ & 0.885 \\
\hline
\end{tabular}

Data are presented as numbers (proportion, \%).

Abbreviations: Group C, control group; Group K, ketamine group; CPSP, chronic postoperative surgical pain; NRS, numeric rating scale.

\section{Figures}




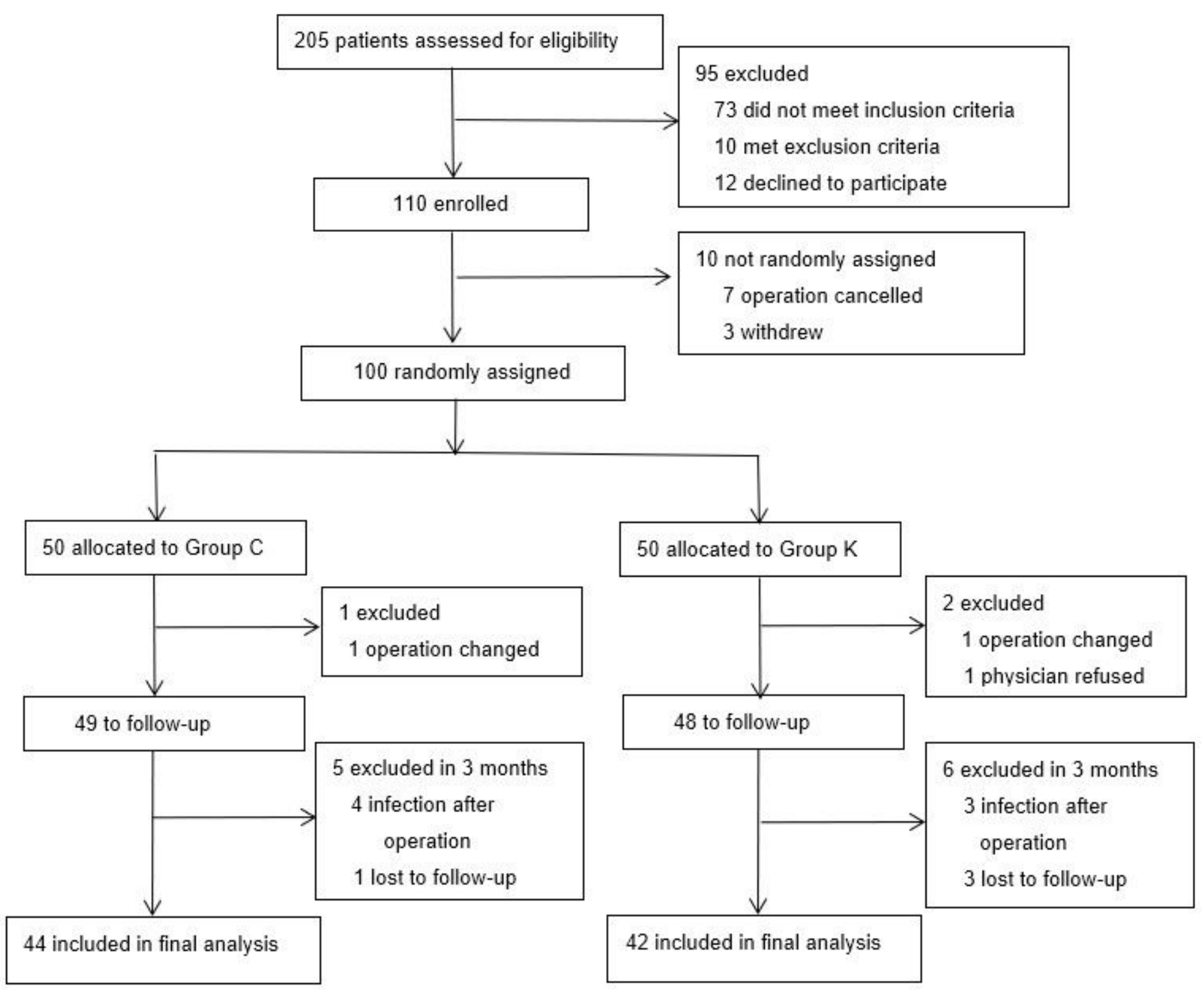

\section{Figure 1}

CONSORT flow diagram of participants

\section{Supplementary Files}

This is a list of supplementary files associated with this preprint. Click to download.

- Additionalfile2.xls

- Additionalfile1.doc 